Alier Forment, M., Casañ, M. J., Piguillem Poch, J., Galanis, N., Mayol, E., Conde, M. Á., \& García-Peñalvo, F. J. (2013). Integration of Google Docs as a Collaborative Activity within the LMS Using IMS BasicLTI. In M. D. Lytras, D. Ruan, R. Tennyson, P. Ordoñez de Pablos, F. J. García-Peñalvo, \& L. Rusu (Eds.), Information Systems, E-learning, and Knowledge Management Research. 4th World Summit on the Knowledge Society, WSKS 2011, Mykonos, Greece, September 21-23, 2011. Revised Selected Papers (pp. 677-683). Berlin, Heidelberg: Springer Verlag. doi: 10.1007/9783-642-35879-1_84

\title{
Integration of Google Docs as a collaborative activity within the LMS using IMS BasicLTI
}

\author{
Marc Alier Forment ${ }^{1}$, Maria José Casañ ${ }^{1}$, Jordi Piguillem Poch $^{1}$, Nikolas Galanis ${ }^{1}$, \\ Enric Mayol $^{1}$, Miguel Angel Conde ${ }^{2}$, Franciso Garcia Peñalvo ${ }^{2}$ \\ ${ }^{1}$ Universitat Politècnica de Catalunya, c/Jordi Girona Salgado 1-3, \\ 08034 Barcelona, Spain \\ ${ }^{2}$ Universidad de Salamanca, Plaza de los caídos S/N. 37008, Salamanca, Spain \\ \{malier,mjcasany,jpiguillem,ngalanis\}@essi.upc.edu \\ \{mconde,fgarcia\}@usal.es
}

\begin{abstract}
Google Docs is a well-known suite of online collaborative tools for document processing, spreadsheets, online presentations, drawing and even quizzes. Google Docs has the potential to become a powerful tool within and LMS course, primarily due to its collaborative qualities. This paper presents an integration that using the IMS BLTI standard turns Google Docs into an engine that powers collaborative learning activities within the Moodle LMS platform.
\end{abstract}

Keywords: VLE, LMS, Interoperability, eLearning, Software as a Service, Cloud Computing, PLEs.

\section{Introduction}

One of the most used acronyms in the world of eLearning is PLE, which stands for Personal Learning Environment. This loose concept shapes a way that long life learners are going to learn. not within a walled garden of the VLE (Virtual Learning Environment or LMS Learning Management System) owned and managed by learning institutions, but as a rather self organized set of sources of information, interaction with peers, learning services, tools, contents and social networks.

This paper presents GDocs-Task, an open source project that embeds the features of Google Docs, as a learning activity within a VLE or LMS course. GDocs-Task works with all the major LMSs in the market, since it is compliant with the interoperability standard IMS BasicLTI.

While this paper is not about PLEs, it has a great deal to do with them. The basic goal of a PLE is how a life long learner should plan, get and organize her learning; she is supposed to learn the necessary knowledge, skills and basic digital competences through her formal learning processes. If these processes only happen within the VLE and the basic set of learning tools implemented on its courses, where content and interaction will remain inside the institution and often will expire shorty after the 
course is finished, the learner will not be able to keep her learning activities nor learn how to build a PLE.

Let's consider this -not so fictional- scenario: a subgroup of students of a class are using tools like Instant Messaging, Wikis, Wave or are participating in a Social Networking site, while -and sometimes for- doing homework. The students engaged in the usage of all these online tools might get better grades or not - some studies say they most likely will -, but they will also learn important skills and become competent in taking advantage of information technologies to access, share information and collaborate with others. However, their teacher may not know about it, because all the information (feedback) she receives is what appears on the LMS's course's logs, and all the tools she is aware of, are the ones bundled in the LMS.

A number of teachers are experimenting with the use of online tools and information services with their students in and out of class. They learn a lot of lessons both from their success cases and their less successful ones. They blog about it, comment on it at conferences and gatherings, tweet about it, etc. But these best practices are not passed along to the big collective of teachers that will, at best, use just the tools provided out of the box by the LMS installed in the institution [1].Another way of facing this challenge is to find out ways to integrate external tools inside the VLE/LMS. This line of work has to consider three main problems:

1. a technical problem of making learning tools and services interoperable,

2. achieving this in a way that has a pedagogical sense and utility for the learner and

3. convincing LMS and tool developers and vendors to comply with the standards proposed.

\section{IMS BasicLTI}

The IMS Global Learning consortium [2] is a well-known international organization working since 2005 in standards towards interoperability and integration of learning services and systems. Major vendors, open source organizations and learning institutions are present on the IMS board of members and enforce the compliance with the standards proposed. Standards like the widely adopted standard for quizzes QTI.

The IMS Learning Tools Interoperability (LTI), developed under supervision of Dr. Charles Severance (creator and former lead developer of the Sakai [3] LMS), focuses on the process on how a remote online tool is installed on a web based learning system. In other words, LTI solves the problem of how the teacher and the student can reach an external application (tool) from within the LMS.

The basic idea of IMS LTI is that the LMS has a proxy tool that provides an endpoint for an externally hosted tool and makes it appear as if the externally hosted tool is running within the LMS. In a sense this is kind of like a smart tool that can host lots of different content. The proxy tool provides the externally hosted tool with information about the user, course, tool placement, and role within the course. In a sense the Proxy Tool allows a single-sign-on behind the scenes and permits an 
externally hosted tool to support many different LMS's with a single instance of the tool.

IMS LTI is a work in progress that has not bet made public yet. But the current IMS Common Cartridge v1.1 standard [4] includes support for BasicLTI. BasicLTI contains the core functionalities that LTI aim for, and its consumer is already implemented in the major LMS open source and proprietary (Moodle, Sakai, OLAT, Angel, WebCT and Blackboard). The authors of this paper have implemented the Moodle 1.9 and Moodle 2.0 consumers for Moodle [5] http://code.google.com/p/basiclti4moodle/. (The Moodle 2.0 consumer is awaiting revision of the Moodle community to be included on the official release of Moodle 2.1. http://tracker.moodle.org/browse/MDL-20534).

\section{Google Docs as a Learning Tool}

Google Docs [6] is a sophisticated cloud computing based set of office tools, that implements a collaborative rich environment that provides the mostly used features of the desktop based office suites.

Google enforces Google Docs for educational purposes [7] because it allows learners to create and edit documents from anywhere and collaborate with multiple people at the same time. Documents are saved online and can be accessed from any computer with an Internet connection. It is also a tool of collaboration, since multiple people can work together on the same document. Google Docs saves automatically, on a regular basis. Each change is tracked as a new revision. The user can see exactly what's been revised, by whom, and when.

If a teacher is invited to share a document with the student she can review, comment, and grade their work at any time, while it is hard for students to get away with unwanted practices when the teacher can review their work at all times. Moreover, the documents can be made public to wider audiences, like parents, or even published directly to blogs.

Google Docs implement a document editor, an online spreadsheet, a presentation tool, a drawing tool, and Goøgle Forms, which the teacher can easily use to create self-grading quizzes [7].

Google Docs is primarily a free service, although there is also a paid version that can be a substitute for using an office suite. Using Google Docs, documents become ubiquitous, so the students can access their documents from school and home. Google makes a strong case about the use of Google Docs for education.

\section{Current integration of Google Docs in Moodle 2.0, not enough}

The latest versions of the most popular LMSs, like Moodle, come with integration with Google Docs. But how do these integrations work? What features do they provide?

Moodle 2.0 integrates Google docs through the repository API. This means that when the user wants to upload a document, he can choose Google Docs as a source 
for this document on Moodle's file picker. So Moodle 2.0 is using Google Docs the same way it uses any other file storage system. Thus we loose all the advantages hinted in section 3: Visibility of the document's creation process, collaboration, possibility for participation by the teacher in the student's document by correcting and hinting at elements, use of quick quizzes created either by the teacher or the students with Forms, tracking and organization of cumulative project data on documents and spreadsheets, and a lot of other, more subtle uses.

\section{GDocs-Task overview}

The GDocs-Task project aims to develop an interoperable software connector that embeds the functionalities of Google Docs as a LMS native activity within the virtual classroom. Let's consider the most simple use case:

1. The teacher sets up a writing activity inside the LMS course. This writing needs to be done with the Google Docs editor. (Fig 1.)

2. When the student enters in the activity, a Document is created within his Google Docs account. This document is shared with the teacher, so the teacher can observe the student's progression through the "Revision history" view, and also can make contributions to the document.

3. When the student considers the assignment is complete he can "deliver" the document within the activity, creating a PDF copy of the current version and sharing it with the teacher. The student will keep the living version of the document and can, if she whishes to, stop sharing the document with the teacher.

4. The teacher can make assessments of the student's activity and send the grades to the LMS course gradebook.

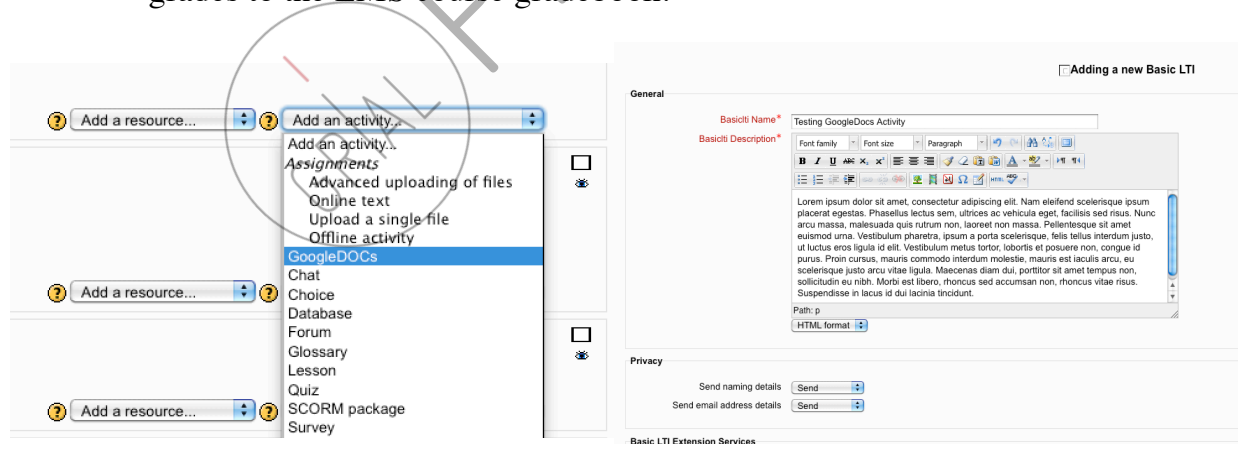


Fig 1. The teacher creates a GoogleDocs activity within Moodle.

More complex use cases include the collaborative editing of documents, where a single document is shared by the whole classroom or group; document workflows where a document needs to be revised by a sequence of students; peer reviewing of documents. These cases are yet to be implemented.

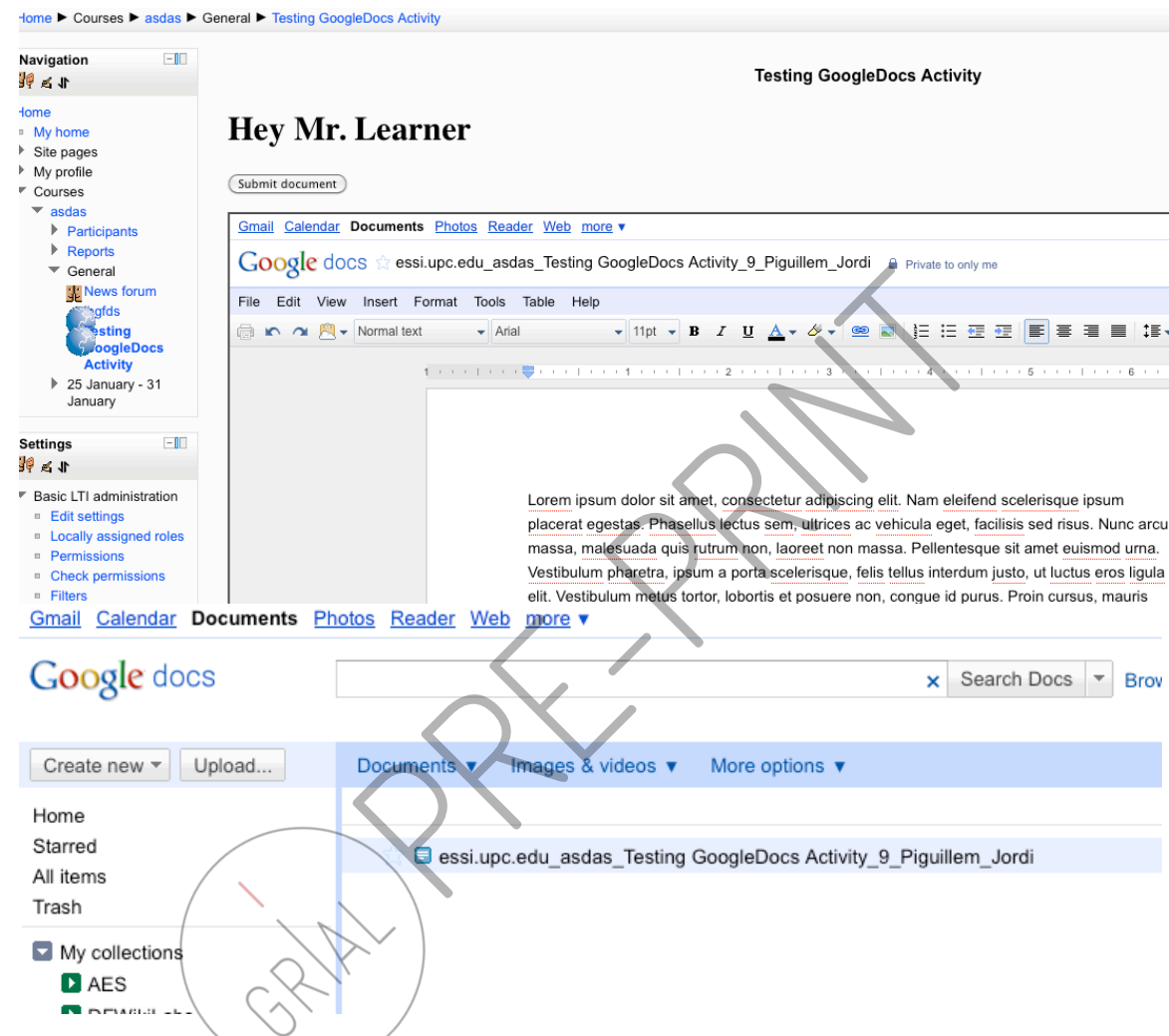

Fig 2. The student can access the document within the LMS or directly in her Google Docs account.

\section{Architecture and security Issues}

\subsection{Architecture}

GDocs-task is a java based web application. It implements the BasicLTI protocol performing as Tool producer. The LMS needs to implement a BasicLTI consumer. Most of the popular LMSs have such a consumer implemented. The LMS consumer 
allows the creation of activities within the LMS courses that are representing the tools outside the LMS. When a user accesses a BasicLTI based activity the consumer interacts with the tool, sending the necessary data back and forth so the user can interact with the tool as if it were an LMS native activity.

GDocs-task also interacts with the webservices API that Google Docs offers to developers. That's how it can perform tasks such as creating and sharing documents, launching the Google Docs user interface and binding it to the LMS.

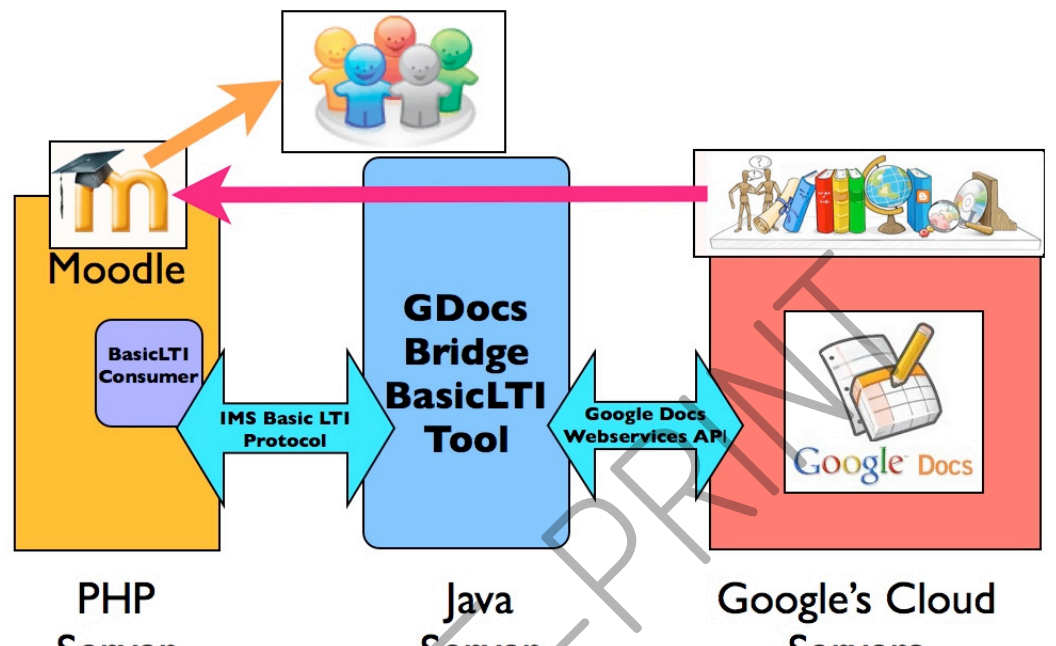

Server

Server

Servers

http://code.google.com/p/gdocs-task http://sushitos.essi.upc.edu

Fig 3. GDocs-Task architecture

\subsection{Security issues}

Privacy and security are two very important issues in this project. We need to be sure that the student's Google account password is revealed to neither GDocs-task nor the LMS. And we need to be sure that Google does not receive information concerning academic matters. Google just needs to handle the data inside the document within the Google Docs terms of service.

GDocs-task, as every BasicLTI tool, needs to be configured by the LMS administrator. The connection between the LMS and the Tool is signed using OAuth [8]. The IMS BasicLTI consumer takes care of this. Additionally, administrator and teacher can limit the information about the student that the LMS is going to provide to the BasicLTI Tools. In the most restrictive case, the student will only be identified by a number to the tool.

Before editing documents and fully using GDocs-task the teacher and the student will be asked by Google to authorize the GDocs-task to access its Google Docs 
service. This is also done using OAuth, and means that the Google account remains a secret between Google and the user.

\section{Future work and conclusions}

The current implementation of GDocs-task is a first step to integrating the full bundle of Google Docs tools: spreadsheets, presentations, painting tool, forms, etc. We need to start experimenting with different ways of wrapping these tools for different educational purposes. We need to work along with teachers to communicate and teach how to use these tools and to learn how to improve them.

More elaborate uses of the architecture require some additions to the IMS BasicLTI standard in form of extensions. We need to follow the IMS process to push our proposals.

Finally other web 2.0 cloud services can be transformed into learning activities and be included in the toolkit that LMS can offer.

\section{Acknowledgements}

A Google research award has funded this project. We thank Google for its support and in particular the Googlers Dr. Max Sengues and Julio Merino.

\section{References}

1. Marc Alier, María José Casany, Jordi Piguillem: Moodle 2.0: Shifting from a Learning Toolkit to a Open Learning Platform. In Technology Enhanced Learning. Quality of Teaching and Educational Reform. ISBN: 978-3-642-13166-0. pp. 1 - 10. Springer Berlin Heidelberg 2010

2. IMS Global Learning Consortium, http://www.imsglobal.org/

3. Sakai Project, http://sakaiproject.org/

4. IMS Common Cartridge v1.1 Specification, http://www.imsglobal.org/cc/

5. Moodle, http://moodle.org/

6. Google Docs, http://docs.google.com

7. Google Tools for Learning, http://sites.google.com/site/ld6edtech/

8. The OAuth 1.0 Protocol RfC. ISSN: 2070-1721. http://tools.ietf.org/html/rfc5849 